\title{
Experiments Directly Demonstrating that the Speed of Light is Independent of the Velocity of the Source: An Experimental Fact or a Misconception
} ${ }^{1}$ V.V. Apollonov, ${ }^{2}$ Yu.P. Voinov

${ }^{1}$ Prokhorov General Physics Institute, Russian Academy of Sciences, ul. Vavilova 38, 119991 Moscow, Russia

${ }^{2}$ Lebedev Physical Institute, Russian Academy of Sciences, Leninsky prosp. 53, 119991 Moscow, Russia vapollo@rambler.ru,voinov@sci.lebedev.ru

Abstract: The issue of the light speed invariance, experimentally studied by E. Aleksandrov et al., seems to be very important. It is shown that Aleksandrov et al. did not take into account a number of factors that lead to a significant difference from the reference data when calculating the speed of light by the method proposed. An accurate analysis of the experiment aimed at measuring the speed of synchrotron radiation allows us to state that it can be equal to $2 \mathrm{c}$. A new experiment is needed in which the synchronization signal from an induction loop introducing an uncertainty is replaced by a signal from the second light detector installed near the radiation point.

Keywords: light speed, theory of relativity, synchrotron emitter.

\section{INTRODUCTION}

In view of the fundamental importance of the light speed invariance postulate, the experimental work of $\mathrm{E}$. Aleksandrov et al. [1] and an analysis of its results in [2] are very significant.

Einstein's special theory of relativity is based, in particular, on the second postulate that "...light is always propagated in empty space with a definite velocity $c$ that is independent of the state of motion of the emitting body" [3]. Invariance of the speed of light makes it possible to explain the experiments of D. Arago, A. Fizeau, and A. Michelson, who observed the independence of the speed of light from the motion of the Earth.

However, there is also the W. Ritz ballistic theory [4--5]. According to the theory, light in vacuum propagates from the source at a speed $c^{\prime}$ equal to the vector sum of the velocities of light $c$ and source $v$ moving relative to the measurement system. The Ritz ballistic theory gave a clear and reasonable explanation of the Michelson-Morley experiment. The ballistic theory also explains other facts that exclude the ether, as well as experiments on the relativistic change in mass. W. Ritz was the first to predict gravitational relativistic effects, explained the secular displacement of the perihelion of Mercury. He derived the formula describing this displacement. A. Einstein came up with his important discoveries much later.

Physicists and astronomers recognize the controversy of previous objections to the Ritz theory, put forward by W. de Sitter on the basis of observations of binary stars. At present, there exist many astrophysical and other experiments that refute the postulate of the constancy of the speed of light [6--14], in particular the radar measurements of Venus and the flight of the Pioneer probes beyond the solar system. From what has been said above, it is clear that the conclusions drawn from the experiment described in [1] are of great importance for science.

We have shown in our paper [2] that E. Aleksandrov et al. [1] did not take into account a number of factors. According to [1], the speed of light calculated by their method gives the value $c=\left(2.85 \times 10^{8}\right) \mathrm{m} \mathrm{s}^{-1}$, which differs from the reference values of the speed of light in a vacuum by $5 \%$. Such a significant discrepancy between the 
Experiments Directly Demonstrating that the Speed of Light is Independent of the Velocity of the Source: An Experimental Fact or a Misconception

velocities and a number of interpretations of the experimental data made us perform a careful analysis of the results of measurements of the speed of light generated by an extremely bright ultrarelativistic synchrotron emitter.

\section{Analysis of the Results of Measuring the SpeEd of Light}

To analyze the results we need to present a brief description of the setup and the results of the experiment from paper [1]. The radiation source is a bunch of electrons moving at a speed close to the speed of light in the Sibir-1 storage ring at the Kurchatov Institute (Fig. 1).

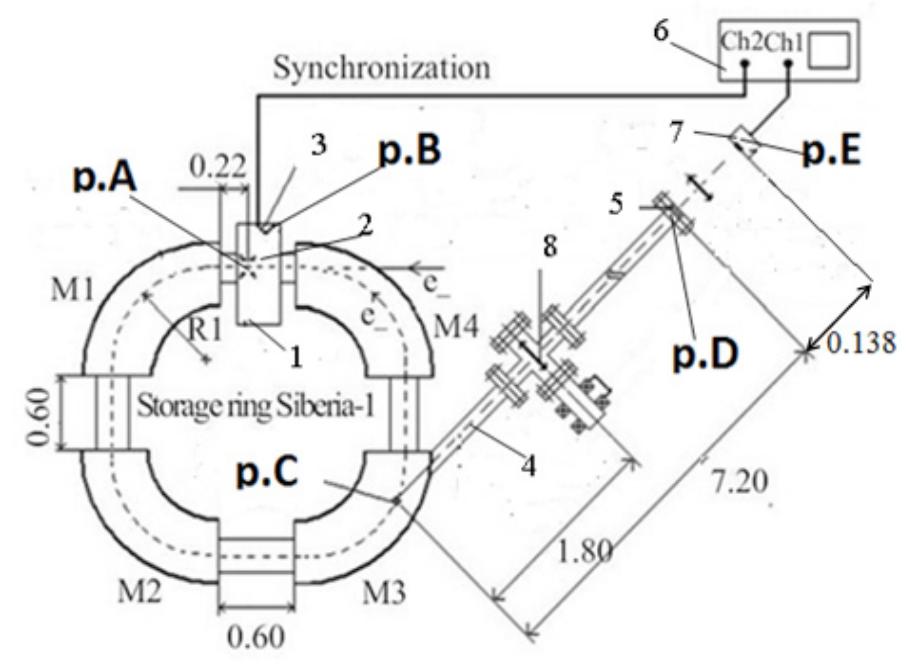

Fig1. Scheme of the experiment: (M1--M4) rotary magnets; (1) resonator; (2) accelerating gap; (3) measuring loop; (4) output channel; (5) output window; (6) oscilloscope; (7) radiation detector; (8) glass plate input--output device. The distances are given in meters.

In Fig. 1, electrons move counterclockwise along a circular path. We introduced additional notations: points $\mathbf{A}$, B, C, D and E, necessary for further analysis:

Point $\mathbf{A}$ shows the location of the accelerating field, which compensates for the radiation losses of the electron beam. A high-frequency voltage (HF), which coincides with the rotation frequency of the electron bunch in the storage ring, $f=34.53 \mathrm{MHz}$, is applied to the accelerating gap at point $\mathbf{A}$.

Point $\mathbf{B}$ shows the location of the measuring loop. The exact position is not indicated by the authors, since it is assumed that this is a standing wave phase of the electric field of the fundamental harmonic of an RF cavity operating at a frequency of $34.53 \mathrm{MHz}$, which is the same for a field in any part of the cavity volume. Therefore, there is no delay in the synchronization signal. However, this is not true. To obtain a standing wave, the resonator length $L$ must satisfy the condition $L \geq \lambda / 2$. At a frequency of the electromagnetic field, $f=34.53$ $\mathrm{MHz}$, the wavelength is $\lambda=868.8 \mathrm{~cm}$. Therefore, the dimensions of the resonator do not satisfy the condition for the formation of a standing wave in it. According to our estimates, the segment length $L_{\mathrm{AB}}$ is $32 \div 38 \mathrm{~cm}$, which at a speed of the electromagnetic wave of $30 \mathrm{~cm} \mathrm{~ns}^{-1}$ leads to a delay of the synchronization signal by $1.07 \div$ $1.27 \mathrm{~ns}$. Generally speaking, the term 'resonator' in this case is used in the sense that the electrons accelerated in this device receive an additional energy due to resonance with the electric HF field in the accelerating gap. The described process has nothing to do with the concept of a resonator of a standing wave, as the authors of the original article suggest. 
Experiments Directly Demonstrating that the Speed of Light is Independent of the Velocity of the Source: An Experimental Fact or a Misconception

Point $\mathbf{C}$ is the starting point for calculating the path length of electrons to point $\mathbf{A}$ and calculating the path length of light to point $\mathbf{E}$.

Point $\mathbf{D}$ is a point to the end of the vacuum tube, near the sapphire window.

Point $\mathbf{E}$ shows the location of the optical sensor.

Point $\mathbf{C}$ is an ephemeral point of contact of the tangential axis of the synchrotron radiation output to the equilibrium electron orbit. The electrons emitted at point $\mathbf{C}$ continue to move counterclockwise up to the accelerating gap at point $\mathbf{A}$, where they are recorded by a measuring loop (point $\mathbf{B}$ ) in the second channel of the oscilloscope. The synchrotron radiation formed at point $\mathbf{C}$ is fed to a silicon PIN-photodiode through the rectilinear channel and is registered in the first channel of the oscilloscope.

To determine the speed of light in the section of the rectilinear channel from point $\mathbf{C}$ to the detector, it is enough to compare this time with the time spent by an electron on the counterclockwise flight from point $\mathbf{C}$ to the initial point A. Calculation is carried out using Fig. 1, which presents the necessary geometric dimensions.

The electron emitted at point $\mathbf{C}$ passes the path of length $L$ to the accelerating gap of the resonator, and the synchrotron radiation formed at the same time in the same point $\mathbf{C}$ moves along the straight line to the receiver at point $\mathbf{E}$.

The path length $L$ of an electron is calculated from the geometry of the device:

$$
\mathrm{L}=\frac{\pi \mathrm{R}}{6}+60 \mathrm{~cm}+\frac{\pi \mathrm{R}}{2}+38 \mathrm{~cm}=52.36+60+157.1+38=307.5 \mathrm{~cm} .
$$

Here, the first term is the length of the trajectory in magnet M 3 when turning by $30^{\circ}$ and $R=100 \mathrm{~cm}$; the second term is a straight line segment between the magnets; the third term is the path length in magnet M4; and the fourth term is the rectilinear segment from the magnet to the accelerating gap (point $\mathbf{A}$ ).

Assuming the velocity of ultrarelativistic electrons to be almost equal to the speed of light, $c=29.98 \mathrm{~cm} \mathrm{~ns}^{-1}$, for the time of flight of an electron along a path of length $L$, we obtain $t_{e}=10.26 \mathrm{~ns}$. (1)

Taking into account the scatter in determining the path length, the error in estimating the time is $\Delta t_{e} \approx \pm 0.3 \mathrm{~ns}$.

The time of flight of the signal from the accelerating gap (point $\mathbf{A}$ ) to the measuring loop (point $\mathbf{B}$ ), proceeding from the earlier estimate of the $L_{\mathrm{AB}}$ dimensions, is $t_{\mathrm{AB}} \sim(1.17 \pm 0.1)$ ns. (2)

Thus, the calculated total signal time of flight in the segment C-A-B from the electron beam is $T_{e}=10.26 \mathrm{~ns}+$ $1.17 \mathrm{~ns}=(11.43 \pm 0.4) \mathrm{ns} .(3)$

The path length $L_{\mathrm{CE}}$ of the light pulse (synchrotron radiation) from point $\mathbf{C}$ to point $\mathbf{E}$ is $L_{\mathrm{CE}}=720+13.8=733.8 \mathrm{~cm}$.

An analysis of the results of the passage of a segment $L_{\mathrm{CE}}$ containing a glass plate showed (see Appendix 1) that the synchrotron radiation velocity does not change when passing through a transparent medium and can be either $c$ or $2 c$. At the same time, a 5\% error in the measurement of the speed of light indicates fundamental inconsistencies in the estimates of the times of passage of various segments of the experimental setup by electrons and radiation.

Let us try a different approach. We assume, in accordance with the Ritz ballistic theory, that the speed of synchrotron radiation (SR) relative to the laboratory (fixed) coordinate system is $2 c$. 
Experiments Directly Demonstrating that the Speed of Light is Independent of the Velocity of the Source: An Experimental Fact or a Misconception

Then the total time for light to travel from the radiation point, point $\mathbf{C}$, to the radiation receiver, point $\mathbf{E}$, is determined by the formula:

$$
T_{S R}=\frac{720+13.8}{60}=12.23 n s
$$

Taking into account the uncertainty of the location of point $\mathbf{C}$, the error in determining $T_{\mathrm{SR}}$ is about $\pm 0.2 \mathrm{~ns}$.

Thus, the calculated lag of the signal from the light pulse with respect to the signal from the electron at the places of their detection (i.e. points $\mathbf{E}$ and $\mathbf{B}$ ) is determined by the difference between expressions (4) and (3):

$$
\Delta T=T_{\mathrm{SR}}-T_{e}=12.23-11.43=0.8 \mathrm{~ns} .
$$

The resulting error in determining the time difference is

$$
0.2+0.4= \pm 0.6 \mathrm{~ns} \text {. }
$$

We will measure the delay time of the light pulse relative to the phase of flight of the accelerating gap by an electron bunch at point $\mathbf{B}$, using the oscillograms presented by the authors in their paper [1].

The point of the counting time of the synchronization channel (channel 2) in the given oscillograms is determined from the condition of action of the autophasing mechanism in the synchrotron.

In the original paper [1], the authors calculated the phase $\phi_{0}$ based on the condition for replenishing the energy losses of an electron in one revolution along an equilibrium orbit. This equilibrium phase corresponds to the time $t=1.16 \mathrm{~ns}$ up to the sinusoid node, i.e. the intersection point with the abscissa axis.

Let us now determine whether the calculated delay $\Delta T=(0.8 \pm 0.6)$ ns corresponds to the measured interval on the oscillograms obtained by the authors. We make preliminary the following remarks.

The sinusoidal curve (2) corresponds to the voltage removed from the measuring loop at point $\mathbf{B}$ of the resonator (Fig. 1).

The loop in the resonator is oriented in such a way that the voltage removed from it is shifted in phase by $180^{\circ}$ relative to the voltage at the accelerating gap of the resonator. Taking into account the fact that the electron charge is negative, the accelerating voltage for it is additionally shifted by $180^{\circ}$ relative to the accelerating voltage for the positive particle. Thus, the total phase shift for an electron is $2 \pi$, i.e. use can be made of the solid curve (2).

Note also that the time of flight from the node of the sinusoid to the accelerating gap is calculated by the authors for a positively charged particle. This follows from the definition of the concept of voltage, i.e. voltage is the work done by an electric field over a positively charged particle. Therefore, the use of the dashed sinusoidal curve by the authors in the calculations is not justified. For the electron, an additional phase shift is equal to $180^{\circ}$, which means that it is necessary to use the solid sinusoidal curve rather than the dashed one.

The oscillograms presented by the authors of [2] show that the thickness of the curves is $\sim 1 \mathrm{~ns}$ according to the optimistic estimate. In addition, the half-periods of the sinusoid are not equal to each other, which is due either to the nonlinearity of the scan or, rather, to the inaccuracy of the ' 0 ' insertion, which introduces an additional error in measuring the time intervals.

From all above said, it follows that measuring $\Delta T=(0.8 \pm 0.6)$ ns on the presented oscillogram is very problematic.

We therefore use the oscillogram of Fig. 2, presented by the authors as an illustration of the achievable accuracy after digital averaging. 


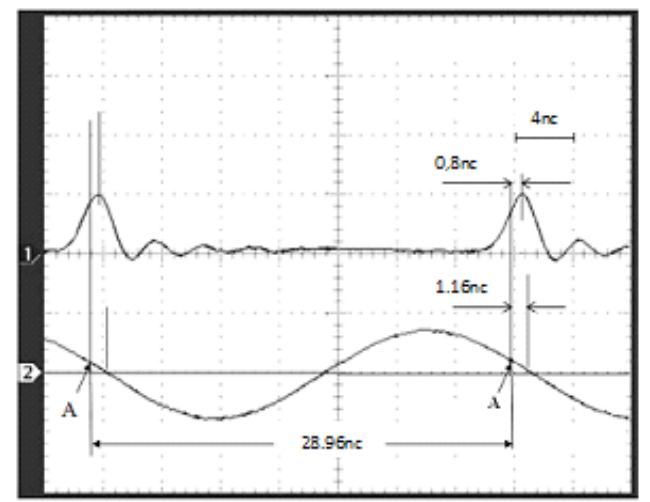

Fig2. Oscillogram after digital averaging, used by us to measure the delay of the light pulse.

Notations 'point $\mathbf{A}$ ' correspond to the instants of time when an electron passes through the accelerating gap in the resonator. These points, as indicated above, are spaced from the nodes at a distance of $1.16 \mathrm{~ns}$. The time interval measured from the given oscillogram from these points to the corresponding maxima of the light signal is $\sim 0.8 \mathrm{~ns}$. The measurement error for this oscillogram is $\sim( \pm 0.2) \mathrm{ns}$. Comparing the delay measured by the oscillogram, $\sim 0.8 \pm 0.2 \mathrm{~ns}$, with a delay calculated from the geometrical dimensions, $\Delta T=0.8 \pm 0.6 \mathrm{~ns}$ (equality 5 ), we see that they agree well within the measurement error.

Since in our calculations we proceeded from the W. Ritz ballistic theory, assuming the SR velocity equal to $\approx 2 c$, we conclude that the results of this experiment confirm the ballistic theory, i.e., the speed of light depends on the velocity of the source. From what has been said above it becomes clear that the high hopes for an unambiguous conclusion drawn from the experiment described in [1] have not been justified.

\section{CONCLUSIONS}

In conclusion, we note that in accordance with our analysis, the results obtained in [1] can confirm the Ritz ballistic theory. In the experiment there is an uncertainty in the choice of the phase of the reference signal (channel 2) on the oscillograms, because the ordinate axis can be chosen directed downward for this channel. To remove this contradiction, it is necessary to conduct an absolutely correct experiment, replacing the synchronization signal from the induction loop introducing an uncertainty by the signal from the second light detector installed near point $\mathbf{C}$.

\section{Appendix 1. Analysis of the Passage of Radiation Through the Glass Plate}

E. Aleksandrov et al [1] presented an analysis of the experiment demonstrating the passage of light through a 7.2-m-long control segment with an open diaphragm and with a diaphragm covered with a $\sim 1$-mm-thick glass plate. Because the time required for the light to travel the control segment is the same for the case with the plate and without it, the authors concluded that "the speed of light emitted by relativistic electrons in a vacuum is equal to the speed of light transmitted through a glass plate in a vacuum, i.e., it does not depend on the velocity of the source. Thus, the experiment refutes the Ritz ballistic theory." However, this consideration does not seem convincing. The equality of the speed of light under the conditions of the experiment with and without a plate indicates only that the speed of the light pulse did not change, i.e. it could be equal to either $c$ or $2 c$.

Following Feynman's ideas [15], we will show how the results of the experiment can be explained. According to Feynman et al. [15]:

a) the total electric field under any physical conditions can be represented as a sum of $\Sigma \overrightarrow{\boldsymbol{E}}$ of the fields from all the charges in the Universe; and 
Experiments Directly Demonstrating that the Speed of Light is Independent of the Velocity of the Source: An Experimental Fact or a Misconception

b) the radiation field of each individual charge is determined by its acceleration, while the acceleration is set taking into account the delay arising due to the finite propagation velocity. Suppose that the W. Ritz ballistic theory is correct. Then, by the propagation velocity is meant the velocity equal to $2 c$.

We are interested in the field $\overrightarrow{\boldsymbol{E}}$ at point D (Fig. 1) at the end of the 7.2-m-long control segment after the light passes through a thin glass plate.

At point $\mathbf{C}$ there is a source of synchrotron radiation, which produces an electric field $\boldsymbol{E}_{\mathrm{s}}$. This field propagates to the right at a speed of $2 c$. According to the above formulated principles, the electric field far from the plate (at point $\mathbf{D}$ ) is represented by the vector sum of the fields of the external source (at point $\mathbf{C}$ ) and the fields of all charges in the glass plate, each field being taken with the corresponding delay. Thus, the field at point $\mathbf{D}$ can be written in the form:

$$
E_{D}=E_{S}+\Sigma E_{\text {ind.charge }}
$$

where $E_{S}$ is the field of an external source (synchrotron radiation); it would coincide with the desired field at point $\mathbf{D}$, if there were no plate.

We recall that the field of each charge does not change in the presence of other charges. Moreover, the field $E_{S}$ propagates with a speed $2 c$, and the vector sum of the fields from the oscillating electrons of the glass plate propagates with a speed $c$. Therefore, the peaks of the registered radiation from $E_{S}$ (synchrotron radiation) and from the glass plate should be in different places on the time scale.

To understand why the peaks from the emission of a glass plate are not observed, let us consider the dynamics of the passage of a synchrotron pulse through this plate.

The field $E_{S}$ of synchrotron radiation, passing through the plate, causes oscillations of charges in the plate. Since the speed of propagation of the field $E_{S}$ in the plate, according to the previously stated provision (b), is equal to $2 c$, it has with a high degree of accuracy the same phase over the entire depth $\Delta z$ of the plate.

This electric field affects each electron in the plate atom and, under the action of the electric force $q E$ they oscillate with the same phase, being in turn new sources of elementary fields having one phase in time and moving to the right along the $z$ coordinate. However, the speed of these fields will already be $c$. Let us consider the radiation dynamics of the intrinsic field of a glass plate (Fig. 3).

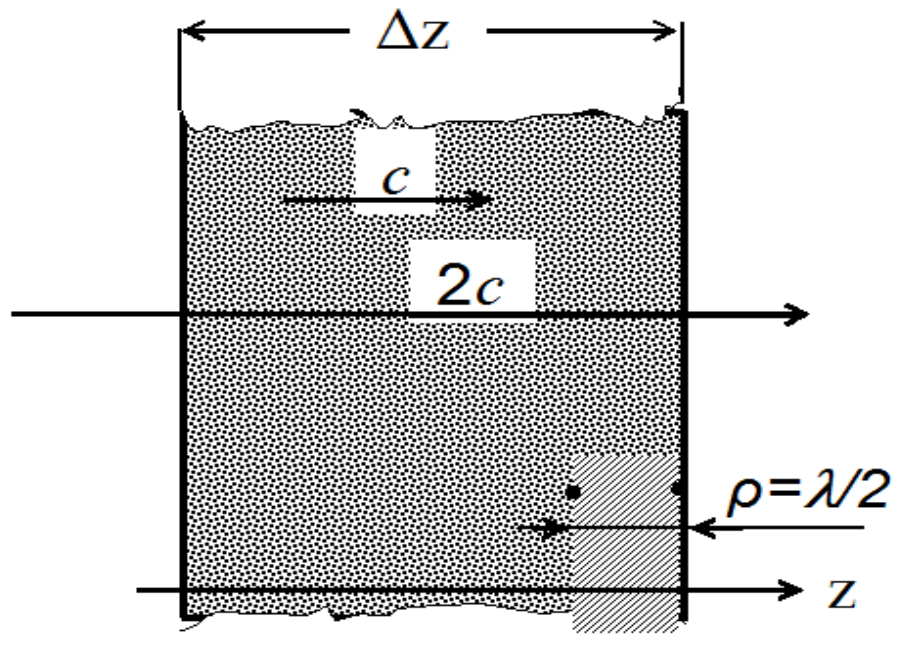

Fig3. Formation of the skin-layer. 
Experiments Directly Demonstrating that the Speed of Light is Independent of the Velocity of the Source: An Experimental Fact or a Misconception

Suppose that at a time $t=0$ the synchrotron field $E_{S}$ flies through the thickness of the plate, $\Delta z$, with a velocity $2 c$. At this moment, all the electrons begin to radiate on the segment $\Delta z$. At a time $t=\rho / c$ (where $\rho$ is the distance from the rear face to the depth of the plate), the phase of the moving intrinsic field of the plate reaching the rear face lags behind the radiation phase at a given point by $\Delta \varphi=\frac{2 \pi}{\lambda}$. If $\rho=\lambda / 2$, the phase difference is $\Delta \varphi=\pi$ and the resultant field of the medium is $E_{a}=0$. For any $\rho_{1} \geq \lambda / 2$ there is an appropriate atom having the ordinate $\rho_{2}=\rho_{1}-\lambda / 2$. Therefore, the fields from the atoms with the coordinates $\rho_{1}$ and $\rho_{2}$ are in anti-phase and are mutually destroyed in the interference. Consequently, the contribution to the intrinsic radiation field of the plate in this particular case is made only by the 'skin layer' with a thickness of $\lambda / 2$ at the rear face of the plate.

This situation is analogous to the case of emission of a diffraction grating, the direction to the emission maxima for which is given by the formula $d \sin \theta=m \lambda$. For our case, the angle $\theta$ is measured from the plane coinciding with the rear edge of the plate. At a distance between the atoms, $d<\lambda$, there is a unique solution for $m=0$, i.e. the grating does not radiate in the $z$ direction.

The maximum value of the intrinsic field of a plate can be estimated from the formula derived by R. Feynman for a glass plate [10]:

$$
E_{a}=-\frac{N \Delta z q_{e}}{2 \varepsilon_{O} c}\left[i \omega \frac{q_{e}}{m\left(\omega_{O}^{2}-\omega^{2}\right)} E_{O} e^{i \omega(t-z / c)}\right]
$$

Assuming below that $\omega>\omega_{0}$, since synchrotron radiation lies in the short-wavelength region of the spectrum, and that only the 'skin layer' contributes to the radiation field of the plate, i.e. $\Delta z \rightarrow \lambda / 2$, we obtain

$$
\left|\frac{E_{a}}{E_{S}}\right| \cong 0.2
$$

Thus, the signal level from the intrinsic field of the plate does not exceed $4 \%$ of the synchrotron radiation field:

$$
\left\langle E_{a}^{2}\right\rangle=0.04\left\langle E_{S}^{2}\right\rangle
$$

and it is not visible on the oscillogram at a given sensitivity of the instrument.

Note that the assumption about the equality of the phases of the oscillating electrons does not affect the final result of the estimates. If we take into account the speed $c$ of the intrinsic field propagation in the plate, then the size of the radiation 'skin layer' will not change.

A similar situation occurs in the segment $L_{\mathrm{DE}}$. Therefore, along the entire segment, from point $\mathbf{D}$ to point $\mathbf{E}$, the speed of light is also $2 c$ and the refractive index $n$ should not be taken into account, which was done in the calculation using formula (4).

\section{REFERENCES}

1. Aleksandrov E.B., Aleksandrov P.A., Zapasskii V.S., et al. Phys. Usp., 54 (12), 1272 (2011) [Usp. Fiz. Nauk, 181 (12), 1345 (2011)].

2. Apollonov V.V., Voinov Yu.P. The Way of Science, No. 3 (25), 10, (2016).

3. Einstein A. Ann. der Phys., Ser. 4, 17 (10), 891 (1905).

4. Ritz W. Ann. Chim. Phys., Ser. 8, 13, 145 (1908). 
Experiments Directly Demonstrating that the Speed of Light is Independent of the Velocity of the Source: An Experimental Fact or a Misconception

5. Ritz W. Arch. Sci. Phys. Nat. de Geneva, 26, 209 (1908).

6. Belopolsky A.A. Astronomicheskie trudy (Astronomical Works) [in Russian] (Moscow: Izd-vo AN SSSR, 1954) (the same on www.luchemet.narod.ru).

7. Batten A.H. Binary and Multiple Systems of Stars (Oxford: Pergamon Press, 1973).

8. Vavilov S.I. Sobranie sochinenii (Collected Works) (Moscow: AN SSSR, 1956), Vol. IV [in Russian].

9. Demin V.N., Seleznev V.P. Mirozdanie postigaya... (Comprehending the Universe...) (Moscow: Molodaya gvardiya, 1989) [in Russian].

10. Wallace B.G. Spectrosc. Lett., 2, 361 (1969).

11. Semikov S.A. Ballisticheskaya teoriya Ritsa i kartina mirozdaniya (The Ballistic Theory of Ritz and Picture of the Universe) (N. Novgorod: Prescontour, 2009) [in Russian].

12. Smul'skii I.I. Elektromagnitnoe i gravitatsionnoe vzaimodeistvie (Electromagnetic and Gravitational Interaction) (Novosibirsk: Nauka, 1994) [in Russian].

13. Tsivinskii S.V. Fizika XXI veka (Physics of the XXI Century) (Moscow: Kompaniya Sputnik, 2007) [in Russian].

14. Sekerin V.I. Teoriya otnositel'nosti - mistificatsiya veka (The Theory of Relativity - The Mystification of the Century) (Novosibirsk: IPP Art-Avenue, 2007) [in Russian].

15. Feynman R., Leighton R., Sands M. The Feynman Lectures on Physics (London: Addison-Wesley, 1963), Vol. 1.

Citation: V.V. Apollonov, Yu.P. Voinov, "Experiments Directly Demonstrating that the Speed of Light is Independent of the Velocity of the Source: An Experimental Fact or a Misconception" American Research Journal of Physics, vol 3, no. 1, 2017, pp. 1-8.

Copyright (C) 2017 V.V. Apollonov, Yu.P. Voinov, This is an open access article distributed under the Creative Commons Attribution License, which permits unrestricted use, distribution, and reproduction in any medium, provided the original work is properly cited. 